\title{
The Impact of Heterogeneity and Uncertainty on Prediction of Response to Therapy Using Dynamic MRI Data
}

\author{
Manav Bhushan ${ }^{1,2}$, Julia A. Schnabel ${ }^{1}$, Michael Chappell ${ }^{1}$, Fergus Gleeson ${ }^{3}$, \\ Mark Anderson ${ }^{3}$, Jamie Franklin ${ }^{3}$, Sir Michael Brady ${ }^{4}$, and Mark Jenkinson ${ }^{2}$ \\ ${ }^{1}$ Institute of Biomedical Engineering (Department of Engineering Science), \\ University of Oxford, $\mathrm{UK}^{\star}$ \\ 2 The Oxford Centre for Functional MRI of the Brain, \\ Nuffield Department of Clinical Neurosciences, University of Oxford, UK \\ 3 Department of Radiology, Churchill Hospital, Oxford, UK \\ 4 Department of Oncology, University of Oxford, UK
}

\begin{abstract}
A comprehensive framework for predicting response to therapy on the basis of heterogeneity in dceMRI parameter maps is presented. A motion-correction method for dceMRI sequences is extended to incorporate uncertainties in the pharmacokinetic parameter maps using a variational Bayes framework. Simple measures of heterogeneity (with and without uncertainty) in parameter maps for colorectal cancer tumours imaged before therapy are computed, and tested for their ability to distinguish between responders and non-responders to therapy. The statistical analysis demonstrates the importance of using the spatial distribution of parameters, and their uncertainties, when computing heterogeneity measures and using them to predict response on the basis of the pre-therapy scan. The results also demonstrate the benefits of using the ratio of $K^{\text {trans }}$ with the bolus arrival time as a biomarker.
\end{abstract}

\section{Introduction}

In the past decade, dynamic contrast-enhanced magnetic resonance imaging (dceMRI) has become a widespread and very effective tool for diagnosis and treatment planning in cancer patients [1. In the case of certain cancers such as breast [2], and cervical cancer [3], the predictive and diagnostic value of dceMRI has already been proven to a large extent. Even though colorectal cancer is the second-biggest cause of cancer-related deaths in Europe, a reliable imaging biomarker is yet to emerge. It is notoriously difficult to constrain motion during dceMRI scans of colorectal tumours, and the importance of motion-correction in estimating pharmacokinetic (PK) modelling has been shown previously in 4, [5]. There are various studies that predict response to treatment by comparing PK parameters before and during treatment, but it is more desirable to predict

\footnotetext{
* Acknowledgements: This work was funded by the CRUK/EPSRC Oxford Cancer Imaging Centre.
} 
response only based on the pre-therapy scan. It is widely acknowledged that intra-tumoural heterogeneity is crucial in this regard [6], [7].

In the analysis of spatial distributions of PK parameters, two principal questions arise: (1) which parameter(s) to consider; (2) what kind of spatial features to look for. The parameters that have been examined most often in the case of dceMRI data, are the transfer coefficients $k_{e p}$ and $K^{\text {trans }}$ (in $\min ^{-1}$ ), that are indicators of vessel permeability (leakiness) and perfusion. Recent literature shows that the bolus arrival time $\left(t_{0}\right)$ is clinically important since it is an indicator of necrosis [8]. In this study, the prognostic value of spatial heterogeneity in $k_{e p}, K^{\text {trans }}$, and a derived biomarker, $r_{k t}=K^{\text {trans }} / t_{0}$ with units $\min ^{-2}$, is investigated. Physiologically, $r_{k t}$ can be likened to a measure of the acceleration experienced by the bolus when it comes into contact with the vasculature. It signifies the ability of the vasculature to respond to the force exerted by the bolus, per unit mass.

In this study, we correct for motion, estimate PK parameters, and then compare some simple measures of heterogeneity. Another crucial aspect of dceMRI data analysis that has been investigated in this paper is the effect of uncertainty in the PK parameter estimates on prediction of response to therapy. The different sources of error and uncertainty in dceMRI data analysis have been analyzed in [9], but the uncertainty has not been integrated into the calculation of heterogeneity in tumours earlier. The model is also parametrized differently in [9], and patient motion is not explicitly taken into account. This study presents a comparison between the predictive values of different measures of heterogeneity calculated with and without taking uncertainty into account.

\section{Methods}

\subsection{Pharmacokinetic Modelling}

In a dceMRI scan, the patient is injected with a low molecular-weight contrast agent (CA), and MRI volumes are acquired at regular intervals after injection. The basic assumption of PK modelling is that the concentration of CA at each voxel in the image space is a function of some matrix physiological parameters $(\theta)$ of the tissue. The changing concetration of CA leads to changes in the MRI signal, which depend on the physiological parameters $\theta$, the MRI acquisition parameters $(\alpha)$, the motion applied during the scan (parametrized by the matrix $T$ ), and a noise process $(\epsilon)$. Thus, the observed data $Y$ is given by:

$$
Y=f(T, \theta, \alpha)+\epsilon
$$

This generic formulation allows any $\mathrm{PK}$ model, $f$, and any noise model, $\epsilon$, to be used. In this study, we have used the Tofts one-compartment PK model [10], and assumed $\epsilon$ to be a Gaussian noise process with mean 0 and precision $\phi$, i.e. $\epsilon \sim N\left(0, \phi^{-1}\right)$. According to the Tofts model, for a given Arterial Input Function (AIF), the concentration of $\mathrm{CA}$ at time $t, C(t)$, can be written as:

$$
C(t)=A I F\left(t-t_{0}\right) \otimes k_{e p} v_{e} e^{-k_{e p}\left(t-t_{0}\right)} \quad\left(\text { for } t>t_{0}\right)
$$


Here $\otimes$ denotes a convolution, $t_{0}$ denotes the time at which the $\mathrm{CA}$ reaches the voxel under consideration, $k_{e p}$ denotes the rate of transfer of $\mathrm{CA}$ from the extra-cellular extra-vascular space (EES) to the blood plasma, $v_{e}$ denotes the proportion of space in that voxel occupied by the EES and $K^{\text {trans }}=k_{e p} \cdot v_{e}$. In order to restrict the number of variables, and to make the analysis more tractable, we have elected to use the Orton (population-averaged) AIF in this study (Model 2 from [11]). We are now interested in extracting the 'true' PK parameter matrix $\left(\theta=\left[K^{\text {trans }}, v_{e}, t_{0}\right]\right)$, which best explain the data $Y$, and the uncertainties associated with these parameters.

\subsection{The Variational Bayes Framework}

In order to accomplish this task, a variational Bayes (VB) framework has been employed for the dceMRI analysis [12. The objective is to maximize the probability of the PK parameters given the data. Thus, using Bayes' rule, we seek to maximize the posterior probability of the parameters given the data:

$$
P(\theta \mid Y, T, \alpha) \propto P(Y \mid \theta, T, \alpha) \cdot P(\theta)
$$

Here, $P(\theta)$ represents the prior probability of the PK parameters, which in this study is assumed to be a multi-variate normal (MVN) distribution, i.e:

$$
P(\theta)=\operatorname{MVN}\left(m_{o}, \Sigma_{0}\right)
$$

Where the matrix $m_{0}=\left[m_{k_{e p}}, m_{v_{e}}, m_{t_{0}}\right]$ represents the prior means of the PK parameters and $\Sigma_{0}$ represents their prior covariance matrix. The VB algorithm takes the mean-field approximation of the posterior distribution, in this case using a MVN for the distribution over the PK parameters. By choosing this conjugate distribution, an iterative update procedure can be derived to find the mean and precision of the distribution. Since the MRI acquisition parameters $\alpha$ remain fixed throughout, and since the VB updates for the PK parameters $(\theta)$ are independent of the motion parameters $T$, we can express $f(T, \theta, \alpha)$ as $f(\theta)$. Now, using Eq (11), due to the assumption of Gaussian noise, we can re-write the likelihood term (for $N$ voxels) from $\mathrm{Eq}(3)$ as:

$$
\begin{aligned}
P(Y \mid \theta, T, \alpha) & = \\
& P(\epsilon=f(\theta)-Y) \\
& \left(\frac{\phi}{2 \pi}\right)^{N / 2} \exp \left(-\frac{1}{2}\left((f(\theta)-Y) \cdot \phi \cdot(f(\theta)-Y)^{T}\right)\right)
\end{aligned}
$$

Now, combining the likelihood term and the prior distributions, we can write the negative $\log$-posterior $(L=-\log P(\theta \mid Y, T, \alpha))$ as:

$$
\begin{aligned}
& L=\frac{1}{2}((\left.f(\theta)-Y) \cdot \phi \cdot(f(\theta)-Y)^{T}\right)-\frac{N}{2} \log \phi \\
&+\frac{1}{2}(\theta-m) \Sigma(\theta-m)^{T}+\text { const }
\end{aligned}
$$

In order to minimize $L$, the function $f(\theta)$ is approximated by a first-order Taylorseries expansion about the mean of the posterior (MVN) distribution to give: $f(\theta)=f(m)+J(\theta-m)$, where $J$ denotes the Jacobian (row vector of partial 
derivatives), calculated at the current estimate of the mean, i.e. $J_{i}=\left.\frac{\partial f(\theta)}{\partial \theta_{i}}\right|_{\theta=m}$. Using this Taylor-series approximation, and following the procedure described in [12, the updates to the mean and covariance matrix for the PK parameters can be computed as:

$$
\begin{gathered}
\Sigma_{n e w}=a J^{T} J+\Sigma_{0} \\
m_{\text {new }} \Sigma_{\text {new }}=a J^{T}\left(k+m_{\text {old }} J\right)+m_{0} \Sigma_{0}
\end{gathered}
$$

Here, $k=|f(\theta)-Y|$, and $a$ is a scalar function of the noise precision updated at each iteration. In addition, after each iteration of the $\mathrm{VB}$ algorithm, we register the raw data to the best-fit model-prediction available at that iteration using a non-linear $\log$ Demons deformation framework [13], and obtain a new estimate of the motion parameters $T$, and thereby a new estimate of the data $Y$.

The final covariance matrix $\Sigma$ can be used to estimate the uncertainties in different derived parameters. In order to estimate the uncertainties for derived parameters $(P)$, such as $K^{\text {trans }}=k_{e p} \cdot v_{e}$ and $r_{k t}=k_{e p} \cdot v_{e} / t_{0}$, we use the Taylor series expansion of $P=g(\theta)=g\left(k_{e p}, v_{e}, t_{0}\right)$, that is: $g(\theta+\partial \theta)=g(\theta)+\nabla g \cdot(\partial \theta)^{T}$, to approximate the variance of $P$ as:

$$
\begin{aligned}
& \operatorname{var}(P)=\quad E\left\{\left(P-P_{\text {mean }}\right)^{2}\right\} \quad \approx E\left\{\left(\nabla g \cdot(\partial \theta)^{T}\right)^{2}\right\} \\
& =(\nabla g) \cdot E\left\{(\partial \theta)^{T} \cdot(\partial \theta)\right\} \cdot(\nabla g)^{T}=(\nabla g) \cdot \Sigma \cdot(\nabla g)^{T}
\end{aligned}
$$

Here $\Sigma$ denotes the covariance matrix of the PK parameters obtained as part of the VB estimation. In this manner, the variance of $K^{\text {trans }}$ can be calculated as:

$$
\operatorname{var}\left(K^{\text {trans }}\right)=\left[v_{e}, k_{e p}\right] \cdot \operatorname{cov}\left(k_{e p}, v_{e}\right) \cdot\left[v_{e}, k_{e p}\right]^{T}
$$

Similarly, the variance of $r_{k t}=k_{e p} \cdot v_{e} / t_{0}$ can be calculated as:

$$
\operatorname{var}\left(r_{k t}\right)=\left[\frac{v_{e}}{t_{0}}, \frac{k_{e p}}{t_{0}}, \frac{-k_{e p} \cdot v_{e}}{t_{0}^{2}}\right] \cdot \operatorname{cov}\left(k_{e p}, v_{e}, t_{0}\right) \cdot\left[\frac{v_{e}}{t_{0}}, \frac{k_{e p}}{t_{0}}, \frac{-k_{e p} \cdot v_{e}}{t_{0}^{2}}\right]^{T}
$$

\subsection{Heterogeneity Metrics}

In this study, we use some standard measures of spatial heterogeneity [6], [7] and compare them with two newly formulated ones. An examination of the dceMRI data available to us showed that most of the tumours had a clearly defined enhancing rim and intra-tumoural vascular structures. It was evident that the enhancing rim and intra-tumoural vascular structures appeared brighter and enhanced sooner than the neighbouring areas. Thus, apart from looking at only the $K^{\text {trans }}$ and $k_{e p}$ maps, we also examined the map of the ratio $r_{k t=} K^{\text {trans }} / t_{0}$ (Fig 1) after additively scaling the values of $t_{0}$ so that the minimum was set to 1. Physiologically, $r_{k t}$ (having units $\min ^{-2}$ ) can be likened to the acceleration experienced by the bolus when it comes in contact with the tissue.

Due to the irregular enhancing vascular structures present in the tumours, we chose to examine the following measures for each parameter map $P$ : 
1. A measure of the fractal nature of the parameter map was estimated by calculating $S_{T}=\frac{1}{2} \sum_{x \in T}\left[\sum_{y \in \Omega_{x}}|P(x)-P(y)|\right] / N_{T}$. Here $T$ represents the tumour region, $N_{T}$ is the number of voxels in the tumour region, and $\Omega_{x}$ represents the six neighbours of the voxel $x$ in $3 \mathrm{D}$.

2 . In order to characterize the rim and other intra-tumoural vascular structures that are visible as sharp, high-value edges in the PK parameter maps, we define the product of $P$ with the gradient map of $P$ as a measure of 'sharpness' $=\frac{1}{2} \sum_{x \in T} P(x)\left[\sum_{y \in \Omega_{x}}(|P(x)-P(y)|)\right] / N_{T}$.

3. The eigenvalues $\left(\left[e_{1}, e_{2}, e_{3}\right]\right.$, where $\left.e_{1}>e_{2}>e_{3}\right)$ of the structure tensor of $P$ can also be used to characterize the linearity or 'planarity' of the parameter map at every position. Since we expect strongly planar parts of the parameter map to be characterized by one dominant eigenvalue, we chose to examine the 'planarity' $=\sum_{x \in T} P(x) \frac{\left(e_{1}(x)-\left(e_{2}(x)+e_{3}(x)\right)\right)}{e_{1}(x)+e_{2}(x)+e_{3}(x)} / N_{T}$.

We calculate each of these three measures for all chosen parameters $\left(r_{k t}, K^{\text {trans }}\right.$ and $k_{e p}$ ), as well as versions that are weighted by uncertainties associated with them (Fig 2), as calculated from the VB algorithm. In order to compute each parameter map with uncertainty, the parameter map was divided point-wise by its corresponding variance (normalized by the average variance across all voxels).

\section{Experiments and Results}

Sixteen patients with locally advanced rectal adenocarcinomas underwent dceMRI scans before treatment with long-course CRT. All the data were acquired on a 1.5T GE scanner using a T1-weighted, gradient-echo, fat-suppressed sequence (LAVA) with $\mathrm{TR}=4.5 \mathrm{~ms}, \mathrm{TE}=2.2 \mathrm{~ms}$ and flip angle of 12 degrees. Four image acquired before the dynamic series, and at different flip angles $(3,9,12$ and 15 degrees), were used to calculate the $T_{10}$ map. The contrast agent (MultiHance) was injected via a peripheral vein and MRI volumes with resolution $1 \times 1 \times 2 \mathrm{~mm}^{3}$ were acquired every 12 seconds for the next 5 minutes.

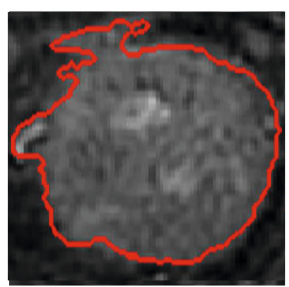

(A)

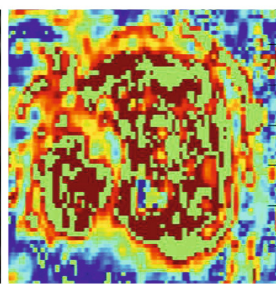

(B)

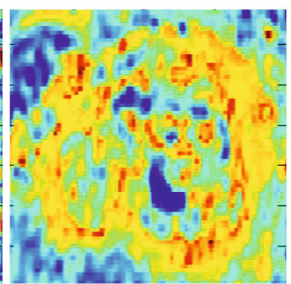

(C)

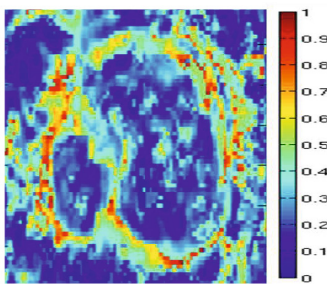

(D)

Fig. 1. The anatomical image showling the outline of the tumour (A), the $k_{e p}$ map in $\min ^{-1}(\mathrm{~B})$, the $K^{\text {trans }}$ map in $\min ^{-1}(\mathrm{C})$ and the $r_{k t}$ map in $\min ^{-2}$ (D) for a rectal adenocarcinoma. 


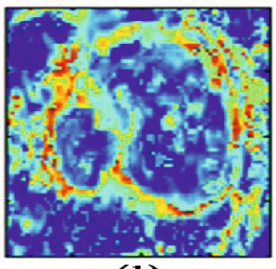

(1)

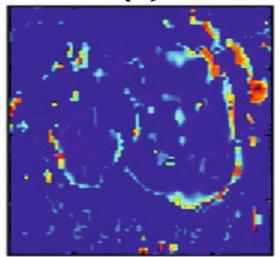

(5)

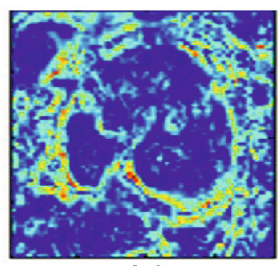

(2)

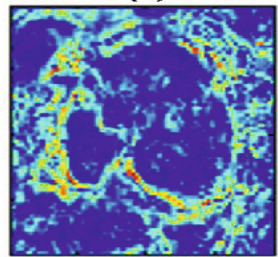

(6)

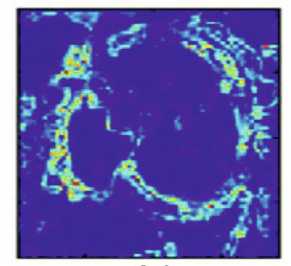

(3)

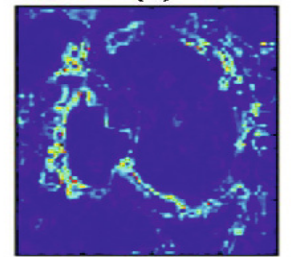

(7)

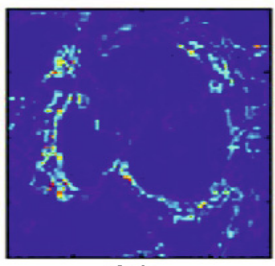

(4)

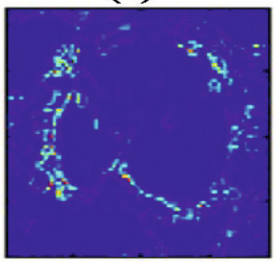

(8)

Fig. 2. The $r_{k t}$ map for a tumour (1), the map showing $S_{T}(2)$, sharpness (3) and planarity (4). The normalized variance map of $r_{k t}(5)$ is then used to weight each of the other three maps: $S_{T}(6)$, sharpness $(7)$ and planarity (8). The display ranges are scaled between the minimum and the maximum values for each parameter map.

An expert radiologist segmented the tumour on high-resolution T2-weighted volumes acquired before the dynamic sequence. This volume was rigidly registered to the first volume of the dynamic scan, and the motion-correction and VB parameter estimation algorithm was applied to a rectangular ROI containing the tumour delineated by the radiologist. In order to compare the predictive values of all the measures of heterogeneity (computed within the segmented tumour), they were compared with the modified Tumour Regression Grade (mTRG) for each patient, calculated after resection at eight weeks [14]. According to the mTRG, eight of the sixteen patients were found to be partial responders $(\mathrm{mTRG}=2)$, while the other eight were found to be non-responders $(\mathrm{mTRG}=3)$.

The ability of each heterogeneity measure to predict response was statistically evaluated in two ways: (1) By computing the $p$-value between the quantities measured for the responder and non-responder groups; (2) Leave-one-out validation (LOOV) - each sample was classified (by fitting a multivariate normal density to each group) using all other samples as the training data, and the average proportion of samples that were mis-classified was recorded (Table 1).

The heterogeneity measures were also compared with the mean and standard deviations of each parameter (across all voxels), in order to evaluate the importance of using spatial distributions as opposed to summary values. The parameter, $r_{k t}$ was found to be the best predictor of response, showing a significant $(p<0.05)$ difference between responders and non-responders for the mean, $S_{T}$, sharpness and planarity measures (Fig 3 ). Furthermore, all the measures of spatial heterogeneity showed consistently better results than the corresponding mean and standard deviation, and would remain significant even after correcting for multiple comparisons. It is also clear from Table 1, that the inclusion of 


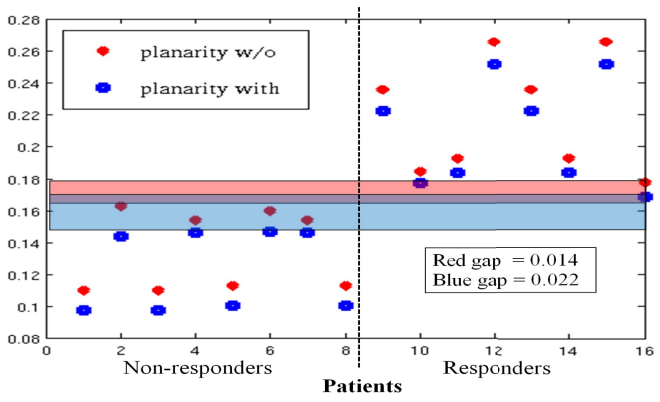

Fig. 3. Plots for the measured 'planarity' in the $r_{k t}$ map across all 16 patients. The red dots indicate the values obtained without incorporating uncertainty, and the red gap is the minimum difference between responders and non-responders for the same. The blue dots indicate the values obtained with uncertainty, and the blue gap is the minimum difference between responders and non-responders for the same.

Table 1. Statistical measures for the difference between the responder $(n=8)$ and non-responder $(n=8)$ groups for five measures using three PK parameters. Each measure is calculated on each parameter map with and without taking uncertainty into account. LOOV represents the proportion of samples mis-classified during leaveone-out validation. The minimum value in each row is indicated in bold.

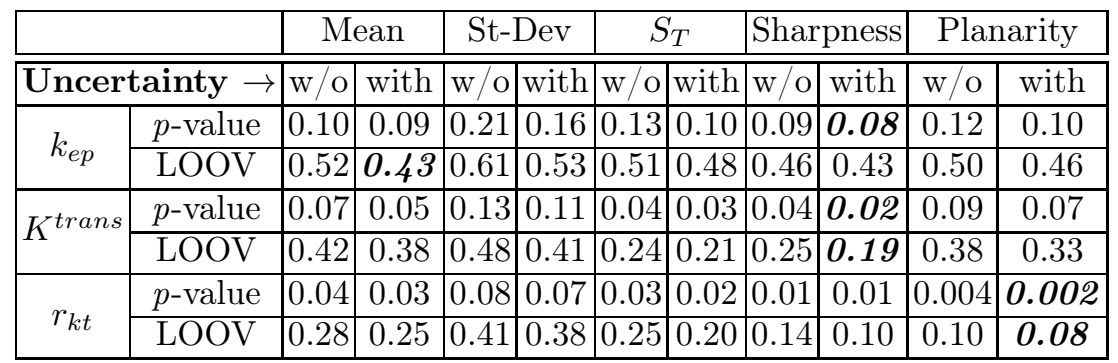

uncertainty in the calculations of different measures consistently improves the ability of each measure to distinguish between responders and non-responders.

\section{Conclusion}

The results indicate that spatial heterogeneity of PK parameters in general, and the new biomarker $r_{k t}=K^{\text {trans }} / t_{o}$ in particular, show very promising potential for predicting response to therapy on the basis of only the pre-therapy dceMRI scan. The results also show that the inclusion of uncertainty in the calculation of heterogeneity measures causes a marked improvement in their ability to distinguish between responders and non-responders to CRT. In future, this model will also be tested with $v_{p}$ as part of the PK model. 


\section{References}

1. Zahra, M.A., Hollingsworth, K.G., Sala, E., Lomas, D.J., Tan, L.T.: Dynamic contrast-enhanced MRI as a predictor of tumour response to radiotherapy. The Lancet Oncology 8(1), 63-74 (2007)

2. He, D., Ma, D., Jin, E.: Dynamic MRI-derived parameters for hot and cold spots: correlation with breast cancer histopathology. Journal of the Balkan Union of Oncology 17(1), 57-64

3. Loncaster, J.A., Carrington, B.M., Sykes, J.R., Jones, A.P., Todd, S.M., Cooper, R., Buckley, D.L., Davidson, S.E., Logue, J.P., Hunter, R.D., West, C.M.: Prediction of radiotherapy outcome using dynamic contrast enhanced MRI of carcinoma of the cervix. Int. J. Radiat. Oncol. Biol. Phys. 54(3), 759-767 (2002)

4. Bhushan, M., Schnabel, J.A., Risser, L., Heinrich, M.P., Brady, J.M., Jenkinson, M.: Motion correction and parameter estimation in dceMRI sequences: application to colorectal cancer. In: Fichtinger, G., Martel, A., Peters, T. (eds.) MICCAI 2011, Part I. LNCS, vol. 6891, pp. 476-483. Springer, Heidelberg (2011)

5. Buonaccorsi, G.A., Roberts, C., Cheung, S., Watson, Y., Davies, K., Jackson, A., Jayson, G.C., Parker, G.J.M.: Tracer kinetic model-driven registration for dynamic contrast enhanced $\{\mathrm{MRI}\}$ time series. In: Duncan, J.S., Gerig, G. (eds.) MICCAI 2005. LNCS, vol. 3749, pp. 91-98. Springer, Heidelberg (2005)

6. Yang, X., Knopp, M.V.: Quantifying tumor vascular heterogeneity with dynamic contrast-enhanced magnetic resonance imaging: a review. Journal of Biomed. and Biotech., 732-848 (2011)

7. O'Connor, J.P.B., Rose, C.J., Jackson, A., Watson, Y., Cheung, S., Maders, F., Whitcher, B.J., Roberts, C., Buonaccorsi, G.A., Thompson, G., Clamp, A.R., Jayson, G.C., Parker, G.J.M.: DCE-MRI biomarkers of tumour heterogeneity predict CRC liver metastasis shrinkage following Bevacizumab and FOLFOX-6. Br. J. Cancer 105, 139-145 (2011)

8. McPhee, K.C.: Delayed Bolus Arrival Time with High Molecular Weight Contrast Agent, an Indicator of Necrosis. Proc. Intl. Soc. Mag. Reson. Med. 20 (2012)

9. Garpebring, A., Brynolfsson, P., Yu, J., Wirestam, R., Johansson, A., Asklund, T., Karlsson, M.: Uncertainty estimation in dynamic contrast-enhanced MRI. Magn. Reson. in Med. 2, 1-11 (2012)

10. Tofts, P.S., Brix, G., Buckley, D.L., Evelhoch, J.L., Henderson, E., Knopp, M.V., Larsson, H.B., Lee, T.Y., Mayr, N.A., Parker, G.J., Port, R.E., Taylor, J., Weisskoff, R.M.: Estimating kinetic parameters from dynamic contrast-enhanced T(1)weighted MRI of a diffusable tracer: standardized quantities and symbols. J. Magn. Reson. Imaging. 10(3), 223-232 (1999)

11. Orton, M.R., D'Arcy, J.A., Walker-Samuel, S., Hawkes, D.J., Atkinson, D., Collins, D.J., Leach, M.O.: Computationally efficient vascular input function models for quantitative kinetic modelling using DCE-MRI. Physics in Medicine and Biology 53, 1225-1239 (2008)

12. Chappell, M., Groves, A., Whitcher, B., Woolrich, M.: Variational Bayesian Inference for a Nonlinear Forward Model. IEEE Transactions on Signal Processing 57, 223-236 (2009)

13. Vercauteren, T., Pennec, X., Perchant, A., Ayache, N.: Symmetric log-domain diffeomorphic registration: A demons-based approach. In: Metaxas, D., Axel, L., Fichtinger, G., Székely, G. (eds.) MICCAI 2008, Part I. LNCS, vol. 5241, pp. 754-761. Springer, Heidelberg (2008)

14. Bateman, A.C., Jaynes, E., Bateman, A.: Rectal cancer staging post neoadjuvant therapy-how should the changes be assessed? Histopathology 54, 713-721 (2009) 